\title{
Ultrasound speed and attenuation in progressive trypsin digested articular cartilage
}

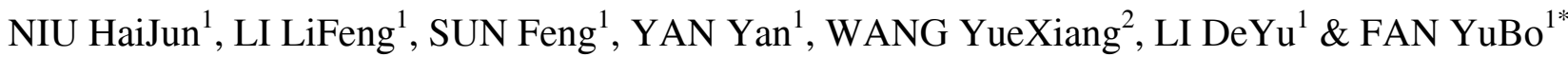 \\ ${ }^{1}$ State Key Laboratory of Software Development Environment, Key Laboratory of Ministry of Education for Biomechanics and Mechanobiology, \\ School of Biological Science and Medical Engineering, Beihang University, Beijing 100191, China; \\ ${ }^{2}$ Department of Ultrasound, People's Liberation Army General Hospital, Beijing 100085, China
}

Received March 10, 2011; accepted September 1, 2011

\begin{abstract}
Subtle changes of articular cartilage (AC) can lead to tissue degeneration and even osteoarthritis (OA). The early degeneration of AC is closely related to a change in proteoglycans (PG) content. The observation of PG is therefore an appropriate way of studying $\mathrm{OA}$ and evaluating the degree of $\mathrm{AC}$ degeneration. In this study, 20 cartilage-bone samples were prepared from normal porcine femoral condyle cartilage and 10 samples were digested over $2 \mathrm{~h}$ using $0.25 \%$ trypsin solution. The dynamic process of PG-digestion was explored using a conventional A-mode ultrasound (US) experimental system with a $10 \mathrm{MHz}$ center frequency. Quantitative acoustic parameters were calculated from ultrasonic radio-frequency echo signals and included US speed (USS), US amplitude attenuation coefficient (UAA) and broadband US attenuation coefficient (BUA). The experimental results showed that the conventional A-mode ultrasound is valuable for tracking the degree of PG-digestion. Histology also confirmed the validity of the ultrasound observations. For every AC sample, the degree of PG-digestion within a given time was different and was affected by individual differences. After two hours of degeneration, USS showed a mean decrease of $0.4 \%(P<0.05)$. UAA was significantly lower after a two-hour PG depletion period (from $(2.45 \pm 0.23)$ to $(2.28 \pm 0.41) \mathrm{dB} \mathrm{mm}^{-1}$ ). BUA showed no significant differences during this process. In conclusion, conventional ultrasound can provide useful information about trypsin-induced progressive PG depletion in AC and can reflect variations of PG content via the quantitative acoustic parameters USS and UAA. The results of this study may be used to identify an indirect indicator of cartilage matrix integrity and OA disease progression.
\end{abstract}

articular cartilage, trypsin degeneration, osteoarthritis, acoustic parameters

Citation: $\quad$ Niu H J, Li L F, Sun F, et al. Ultrasound speed and attenuation in progressive trypsin digested articular cartilage. Sci China Life Sci, 2011, 54: 1029-1035, doi: 10.1007/s11427-011-4235-1

Articular cartilage (AC) is a weight-bearing tissue covering the bony ends of articulating joints. It provides joints with excellent lubrication and has anti-wearing characteristics, and thus maintains a smooth and efficient force-bearing system for the body [1-3]. AC is a multi-phase hydrated mixture composed of proteoglycan (PG; 5\%-10\%), collagen $(10 \%-20 \%)$, and water $(60 \%-80 \%)$ [2-5]. The different components of the tissue are inter-related and contribute to the achievement of its structural integrity.

*Corresponding author (email: yubofan@buaa.edu.cn)
The early stage of osteoarthritis (OA) is asymptomatic. The earliest signs of OA include loss of PG content in AC and disruption of the superficial collagen network [6-8]. A change in PG content and distribution is a continuous process that precedes other components during degeneration. Therefore, evaluating the degree of $\mathrm{AC}$ degeneration based on the observation of PG is particularly appropriate for the study of OA. The changing PG content and distribution is an important indicator of cartilage matrix integrity and disease progression.

Unfortunately, current methods of $\mathrm{PG}$ evaluation in vivo 
require a biopsy on patients, which is an invasive procedure. Several different imaging techniques, such as X-ray computed tomography (CT), magnetic resonance imaging (MRI), optical coherence tomography (OCT), and electromechanical evaluation (EME), have also been used to evaluate the degree of AC lesions [8-14]. CT arthrography is insensitive to subtle changes in PG content $[8,9]$. MRI is currently the most promising method of imaging $\mathrm{AC}$ in vivo. However, large equipment, high costs, and relatively long imaging times limit its use [10-12]. OCT and EME techniques are still immature [13,14].

Because of its microscopic resolution, high frequency ultrasound (HFU) may provide more information about the morphological, acoustical and mechanical properties of soft tissues $[15,16,17,18]$. HFU has been used in the study of cartilage maturity [19], cartilage morphology [20-24], cartilage repair and quantitative evaluation of tissue properties $[25,26]$. Combined with indentation and compression technology, it can even be used to measure the tissue elasticity of AC $[27,28,29,30]$. To identify and characterize the degree of AC degeneration, many studies have investigated the inter-relation between acoustics parameters and component content of AC based on HFU and found that PG-degeneration may lead to decreases of ultrasound speed, increases of attenuation or change of other acoustic parameters [17,26,30,31-37]. These results all demonstrated that $\mathrm{HFU}$ is useful in the study of AC degeneration. However, in clinical diagnosis, the frequency range of conventional ultrasound systems is low (in general no more than $15 \mathrm{MHz}$ ) except for some specialized applications (for example, ophthalmic ultrasound). As a result, the application of HFU is still limited because of its rapid attenuation and low penetration ability in tissue.

In the laboratory, enzyme digestion is a typical method to experimentally induce the depletion or decomposition of one or more components of AC to simulate pathological changes. Trypsin has been applied to digest PGs in the animal and in human AC models of OA [33]. This study aimed to observe the dynamic process of $\mathrm{PG}$ digestion with a conventional A-mode ultrasound experimental system with a 10 $\mathrm{MHz}$ center frequency. Quantitative acoustic parameters were calculated from ultrasonic radio-frequency echo signals, and included US speed (USS), US amplitude attenuation coefficient (UAA) and broadband US attenuation coefficient (BUA). Histological images were used to verify the degree of PG-depletion. The results of the study may provide some valuable information for the early diagnosis of AC diseases.

\section{Materials and methods}

\subsection{Specimen preparation}

Twenty fresh femoral condyles of mature porcine were selected from a slaughterhouse. The AC cover on the femoral condyle was inspected and no apparent lesions were found. Before conducting the experiment, femoral bone was removed, leaving only cartilage and subchondral bone as samples. Two cartilage-bone samples were prepared from the lower medial side of each condyle (Figure 1). One sample was treated as a control and the other was digested with trypsin. Each AC sample with a bone layer was approximately $5 \mathrm{~mm}$ thick, and the thickness of the cartilage layer was $(1.57 \pm 0.56) \mathrm{mm}($ mean $\pm \mathrm{SD}, n=20)$. The samples were stored at $-20^{\circ} \mathrm{C}$ condition until used for experiments.

\subsection{Ultrasound system}

Figure 2 shows a depiction of the ultrasound system used for experiments. The system was composed of the following components: a broadband pulse transmitter/receiver (Model 5800, Olympus, USA), a $10 \mathrm{MHz}$ center frequency focused ultrasound transducer with a focal length of $39.37 \mathrm{~mm}$, bandwidth 6.91-12.14 at $-6 \mathrm{~dB}$ (Panametrics, USA), a computer with a $400 \mathrm{MHz} 12$ bits A/D converter (CompuScope 12400, Gage, Canada). All ultrasound echo signals reflected from the specimens were collected by the transducer and were band-pass filtered (1-35 MHz), then digitized by the $\mathrm{A} / \mathrm{D}$ converter to be saved in the computer for further analysis.

\subsection{PG digestion experiment}

Before conducting experiments, samples were unfrozen in physiological saline for $2 \mathrm{~h}$ at $25^{\circ} \mathrm{C}$ condition and then placed in a container with $0.25 \%$ trypsin solution. The tem-

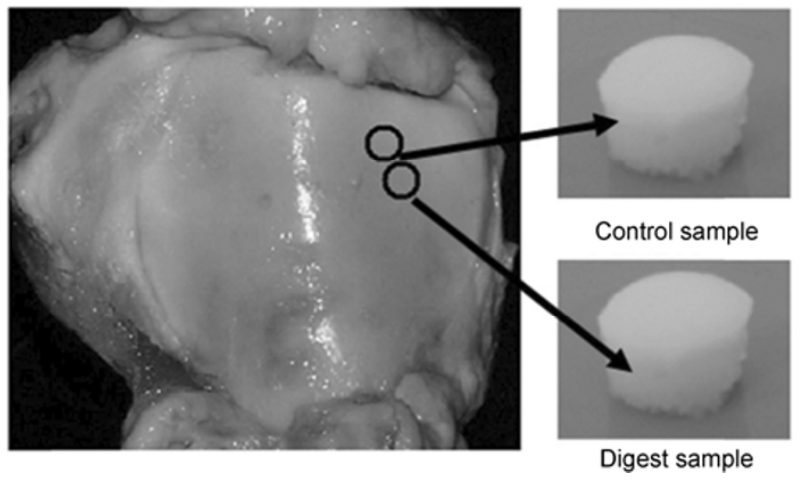

Figure 1 Articular cartilage specimen preparation.

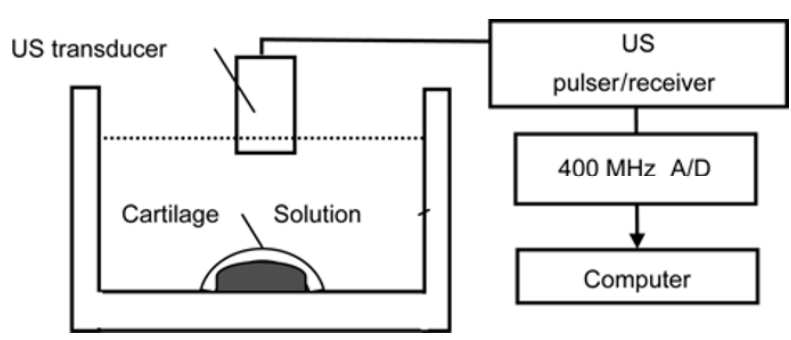

Figure 2 A-mode ultrasound experimental system for AC measurement. 
perature of the solution was controlled at approximately $25^{\circ} \mathrm{C}$ throughout the experiment. The specimen was monitored with the ultrasound system during the progressive PG digestion over $2 \mathrm{~h}$. The focal point of the transducer was placed approximately at the middle layer of the cartilage. Ultrasound echoes were recorded every $10 \mathrm{~s}$ with a 200 $\mathrm{MHz}$ sample rate with commercial software (v. 3.0, Gage, Canada). The data were processed and analyzed off-line using Matlab (v. 7.0, Mathworks, USA).

\subsection{Histological analysis}

After the ultrasound measurements, all control and trypsindigested samples were fixed in $10 \%$ buffered formalin solution, decalcified in an EDTA solution, and then embedded in paraffin. A $5 \mu \mathrm{m}$ thickness longitudinal section from the site near the ultrasound measurement point was obtained and stained with safranin O. In optical micrographs, red color stained by safranin $\mathrm{O}$ indicated the content of the PG component.

\subsection{Acoustic parameters}

The most important ultrasound parameters that have been used for evaluating cartilage degeneration are ultrasound speed (USS) and attenuation (UAA and BUA). To estimate these parameters, ultrasonic radio frequency signals reflected by the cartilage surface and backscattered from its internal matrix were processed in the time and frequency domain. In this study, the relative change of USS was calculated. The initial USS was predetermined as $1630 \mathrm{~m} \mathrm{~s}^{-1}$ $[29,34]$. The thickness of the specimens was calculated according to this value, and the thickness was assumed to not vary during the experiment. This hypothesis is reasonable according to our previous research because the salt concentration of the solution is invariant throughout the experiment. The time of ultrasound flight in the tissue is the period for a round trip between the upper interface (solution-cartilage) and the lower interface (cartilage-subchondral bone).

The ultrasound attenuation coefficient can be calculated in the time domain and frequency domain. Here, two commonly used attenuation coefficients: amplitude attenuation

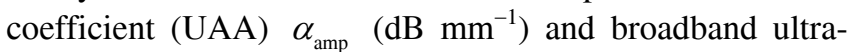
sound attenuation coefficient (BUA) $\left(\mathrm{dB} \mathrm{MHz}{ }^{-1}\right)$ were calculated using the following equations:

$$
\begin{gathered}
\alpha_{\text {amp }}=\frac{10}{h} \log _{10} \frac{A_{1}}{A_{2}}, \\
B U A=\frac{\mathrm{d}}{\mathrm{d} f}\left[10 \log _{10} \frac{A_{1}(f)}{A_{2}(f)}\right],
\end{gathered}
$$

where $A_{1}$ and $A_{2}$ are the peak-to-peak amplitudes of the upper interface echo and the lower interface echo, respectively. $A_{1}(f)$ and $A_{2}(f)$ are the amplitude spectra of these echoes, and $h$ is the thickness of the specimen. The region of interest (ROI) of every echo was chosen according to the computed signal envelope by the Hilbert transform, and rectangular $0.25 \mu$ s windows were centered on the minima signal points that were near the maxima in the envelope of the interface echoes [38]. $A_{1}(f)$ and $A_{2}(f)$ were obtained by a fast Fourier transform of the ROI data. For a more detailed description of ultrasound attenuation, the reader is referred to a related reference [35].

\subsection{Statistical analysis}

Statistical analyses were conducted with SPSS software (v. 17). All values in the text are presented as mean \pm SD. In light of the small number of samples, nonparametric analysis was used in this study. The Mann-Whitney U test was used to evaluate the statistical difference in USS, UAA and BUA of the cartilage samples before and after trypsin digesting.

\section{Results}

Ultrasound echo signals obtained from digested AC specimens are shown in Figure 3. The horizontal axis represents the time of flight in the specimen, and the vertical axis is the amplitude of the ultrasound echo signal. The upper interface (solution-cartilage) and the lower interface (cartilage-subchondral bone) can be discerned clearly in ultrasound echo signal.

Figure 4A shows ultrasound echo signals of the AC specimen at several different times and Figure 4B shows Mmode ultrasound images of the digested AC. From the ultrasound echo signals, an obvious echo at the interface between the digested and undigested tissues was observed and was deeper in the tissue with the trypsin penetrating into the cartilage tissue. The echoes from the digested-undigested interface are circled in the RF signals (Figure 4A). These echo signals gave rise to the inclined trace observed in M-mode image. This downward trend is indicated by the

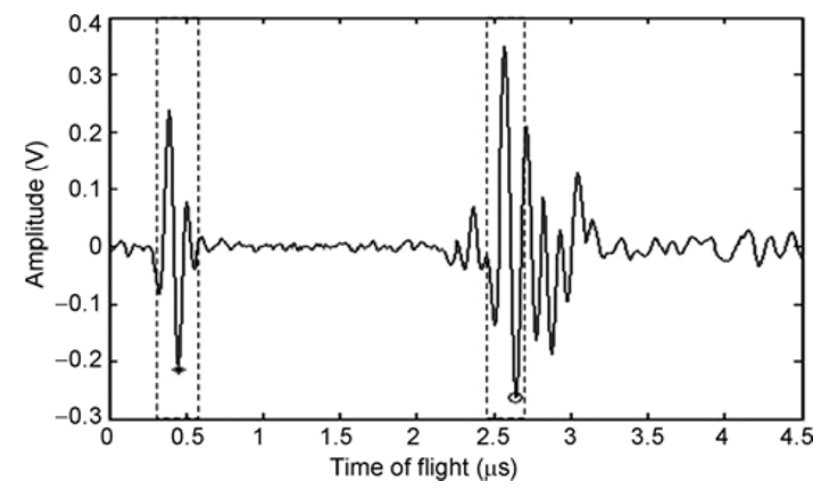

Figure 3 The ultrasound echo signals propagated in $\mathrm{AC}$ were obtained using the ultrasound experimental system. Each box indicates the selection of the region of interest (ROI). 

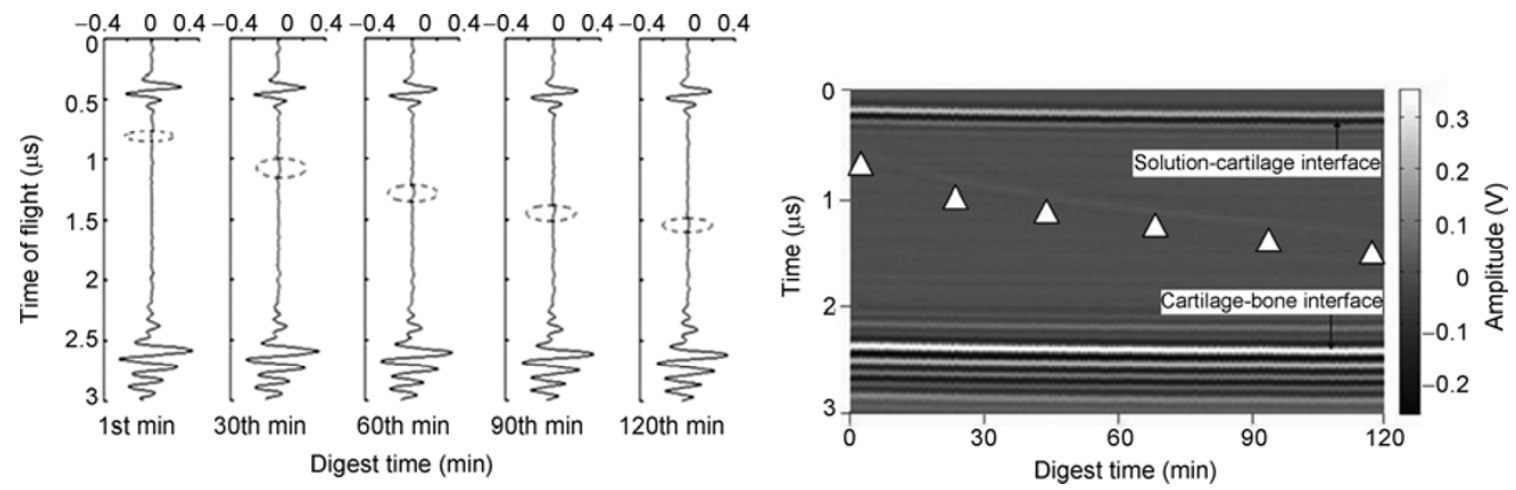

Figure 4 Ultrasound RF echo signals at several different times (A) and M-mode ultrasound image of digested AC (B), where the white triangles indicate the interface between the digested and undigested tissues.

white triangles in M-mode image shown in Figure 4B.

Figure 5A shows the change of the digestion depth (percentage of total specimen thickness) with trypsin treatment time for 10 tested specimens. Figure 5B shows the relationship between digestion depth and trypsin treatment time. The error bars are the standard deviations of the digestion depth for all specimens. Here, the digestion depth was calculated by normalizing the specimen thickness. A secondorder polynomial function was used to fit this relationship $\left(R^{2}=0.990\right)$. The average digestion depths at different times can be estimated based on the polynomial function.

The comparisons between M-mode ultrasound images and histological section images in terms of digestion depth are shown in Figure 6 for two typical specimens. Figure 6 represents the histological section of a specimen of undigested cartilage (control sample) stained with safranin $\mathrm{O}$ (Figure 6A), the M-mode data showing the complete digestion process from the cartilage surface to the bone (Figure $6 \mathrm{~B})$, and the histological section of this completely digested sample stained with safranin O (Figure 6C).

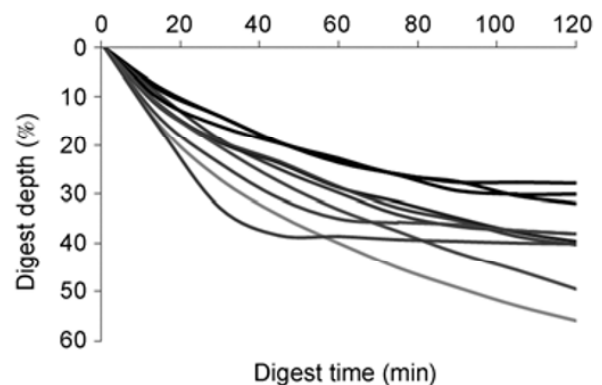

The quantitative ultrasound parameters USS, UAA and BUA were measured for the articular cartilage samples at different digestion times and the results are shown in Table $1($ mean \pm SD). These parameters are also shown graphically in Figures 7 and 8.

Figure 7 shows the change of USS during trypsin digestion of AC. The error bars in the figure represent the SD among the results of all the specimens. A second-order trend line is shown in the figure. Statistically significant changes $(n=10, P<0.05)$ in USS after trypsin digestion were found in porcine AC. The magnitude of USS decreased with the depletion of PG content. After two hours of trypsin digestion, the mean change in USS was $6.6 \mathrm{~m} \mathrm{~s}^{-1}$. The speed of sound in porcine AC was significantly related to tissue degeneration (i.e., the change of the PG content). In all of the experimental processes, no changes in USS were observed in the trypsin solution.

Figure $8 \mathrm{~A}$ and $\mathrm{B}$ shows the changes of ultrasound amplitude attenuation and broadband attenuation in progressive trypsin-digested AC, respectively. The second-order trend

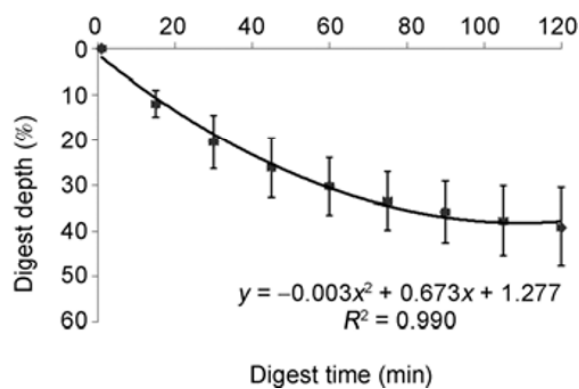

Figure 5 The transient digestion depth as a function of time for the 10 specimens (A) and nonlinear relationship between the digestion depth and the digestion time (B).

Table 1 Ultrasound speed (USS) and attenuation coefficients (UAA and BUA) (mean \pm standard deviation) measured for articular cartilage samples

\begin{tabular}{|c|c|c|c|c|c|c|c|c|c|}
\hline \multirow{2}{*}{ Parameters } & \multicolumn{9}{|c|}{ Digestion time (min) } \\
\hline & 0 & 15 & 30 & 45 & 60 & 75 & 90 & 105 & 120 \\
\hline $\operatorname{USS}\left(\mathrm{m} \mathrm{s}^{-1}\right)$ & $1630.00 \pm 0$ & $1628.12 \pm 3.23$ & $1626.77 \pm 4.75$ & $1625.29 \pm 6.12$ & $1624.56 \pm 8.57$ & $1624.57 \pm 9.66$ & $1623.88 \pm 9.93$ & $1623.59 \pm 10.18$ & $31623.36 \pm 12.26$ \\
\hline UAA & $2.450 \pm 0.238$ & $2.406 \pm 0.255$ & $2.379 \pm 0.276$ & $2.374 \pm 0.291$ & $2.328 \pm 0.337$ & $2.324 \pm 0.362$ & $2.307 \pm 0.384$ & $2.294 \pm 0.395$ & $2.283 \pm 0.413$ \\
\hline BUA & $0.012 \pm 0.149$ & $0.028 \pm 0.143$ & $0.032 \pm 0.150$ & $0.015 \pm 0.152$ & $0.016 \pm 0.148$ & $0.012 \pm 0.146$ & $0.027 \pm 0.141$ & $0.031 \pm 0.140$ & $0.011 \pm 0.139$ \\
\hline
\end{tabular}




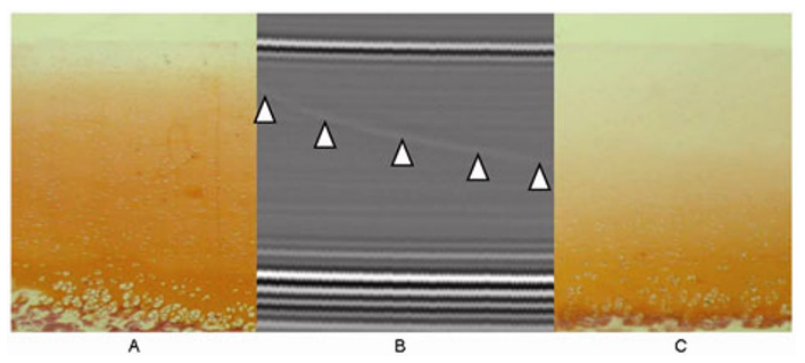

Figure 6 Comparison of M-mode images with histological sections for digested cartilage. A, Histological sections of the control undigested samples stained with safranin O. Fuscous areas indicate high PG concentration and light areas show low PG content. B, M-mode images showing the digestion process of the cartilage sample. C, Histological sections from the digested sample. The white triangles in $\mathrm{B}$ indicate the interface between the digested and undigested tissues.

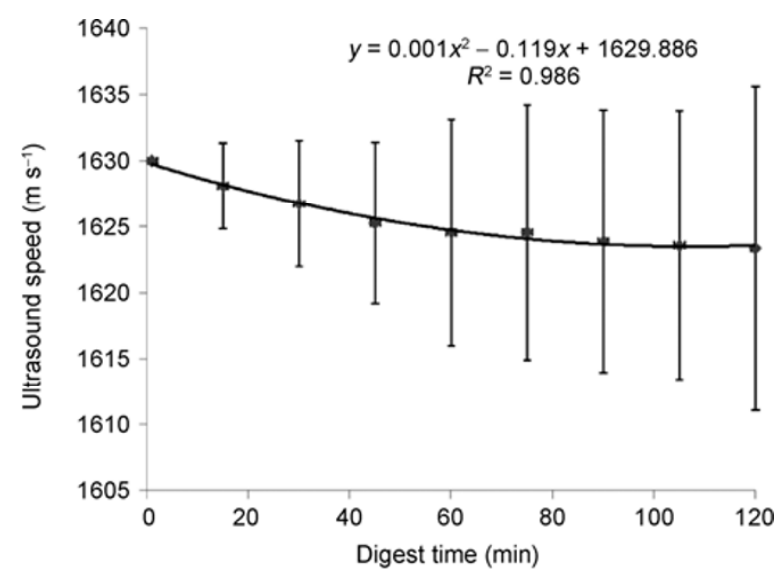

Figure 7 The change of USS in progressive trypsin-digested AC. The presented data show the average of all the specimens and the error bars indicate the standard deviation. It was demonstrated that the USS was significantly related to tissue degeneration (i.e., the change of the PG content).

line is shown in Figure 8A and the first order line is shown in Figure 8B. Amplitude attenuation showed a significant linear correlation with the tissue degeneration of AC (i.e., the change of the PG content) $(n=10, P<0.01)$. The mean value of the ultrasound amplitude attenuation coefficient decreased with the depletion of PG content. After two hours

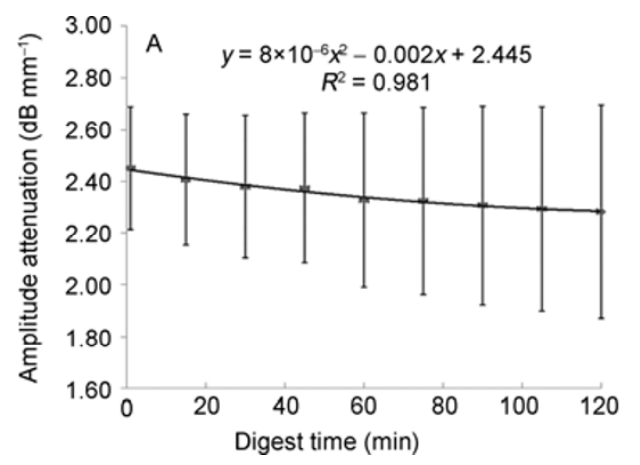

of trypsin digestion, the mean change in the ultrasound amplitude attenuation coefficient was $0.167 \mathrm{~dB} \mathrm{~mm}^{-1}$ (range from 2.45 to $2.28 \mathrm{~dB} \mathrm{~mm}^{-1}$ ). However, for BUA, the mean values of the samples at different time points ranged from 0.011 to $0.032 \mathrm{~dB} \mathrm{MHz}^{-1}$. No significant differences were detected in BUA between the start and end of the PG depletion experiment.

\section{Discussion}

Ultrasound has been suggested a potentially valuable tool for the identification and characterization of the degree of AC degeneration. This study investigated the changes of ultrasound speed and attenuation in progressive trypsindigested porcine AC specimens using a conventional A-mode ultrasound with $10 \mathrm{MHz}$ center frequency, and quantified by the parameters USS, UAA and BUA.

The results of the experiment showed that the digestion progress of the AC specimens by trypsin can be observed with conventional ultrasound. From the A-mode ultrasound waveforms (Figure 4A), a series of small echoes, which formed a light trace with a downward trend in the M-mode ultrasound image as the trypsin penetrated into AC tissue and depleted PG content (Figure 4B), were observed at the interface between the digested and undigested tissues. This observation is consistent with previous studies based on high frequency ultrasound [26,31]. The comparison between M-mode ultrasound images and histological images in terms of digestion depth also confirmed the validity of the ultrasound observation (Figure 6). The light trace formation is the result of sound impedance differences increase between the digested and undigested tissues.

Figure 5 shows the relationship between digestion depth and trypsin digestion time. For different AC specimens, the digestion depth and the digestion trend are also different for the same digestion time. These differences were induced by individual differences of the specimens. However, for a given AC specimen, the digestion depth and the digestion trend can be roughly estimated using a second-order fitted polynomial function.

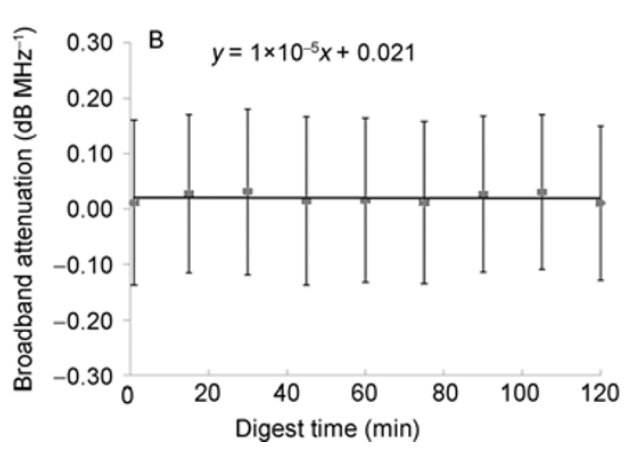

Figure 8 The change of ultrasound amplitude attenuation and broadband attenuation in progressive trypsin-digested AC. The presented data show the average of all the specimens and the error bars indicate the standard deviation. The solid line is the second order trend line of the ultrasound attenuation. 
From Figure 5, the speed of digestion was observed to decrease as the depth of tissue increased. This tendency is related to the distribution of the PG content and it also appeared in studies using high frequency ultrasound by Wang et al. [31,35]. This result implies that the digestion of trypsin depends on the concentration of PG at different depths in the tissue. More trypsin is needed to digest the PG that is deep in the tissue than PG at the superficial layer.

USS is an important parameter in the measurement of ultrasound tissue characteristics. Although there is some variability in reported findings, most studies have shown that USS in AC is related to the composition and structure of the tissue. A decrease in USS, either large or small, has been observed to occur after enzymatic degradation of PG $[8,20,27,34,37]$. These results support the hypothesis that increased water content in the process of PG degeneration would change the USS in cartilage towards that of water, i.e., USS should decrease. The reason is that the compressibility of AC tissue increases with PG degradation, and the high compressibility of the tissue leads to the decrease in USS. The result obtained in the present study is consistent with previous results. However, the decrease of USS is minor, compared with the decrease of $2 \%$ reported by Joiner $e t$ $a l$, and a mean decrease in USS by $0.4 \%(n=10, P<0.05)$ was observed in porcine cartilage samples after two hours of PG depletion [27]. This result was probably induced by a short degeneration time, low enzymatic solution concentration and low experiment temperature.

The ultrasound attenuation is another important characteristic in the study of tissue properties based on ultrasound. The ultrasound attenuation coefficient is used to describe the energy loss of an ultrasound wave as it propagates through the tissue. Pulse energy is lost through a variety of interactions. In this study, it was apparent that the ultrasound amplitude attenuation decreased with enzymatic degradation of PG. Amplitude attenuation coefficients were correlated positively with the US speed $(P<0.01)$. However, the BUA coefficient is non-sensitive to PG degeneration, so no significant differences were detected in BUA between the original samples and the two-hour degenerated samples. This result is consistent with the results reported by Nieminen et al. [34]. However, many consider that the attenuation of ultrasound may increase because of PG degeneration that lowers specimens' density and increases water content of tissue. The digesting process and related effects are very complicated. Aside from the changes of PG content and matrix structure, many other factors such as additional increase in water content in sample preparation, trypsin solution concentration, and experiment temperature, may cause changes in the US attenuation coefficient towards a different direction. These factors should be considered carefully in future studies.

In conclusion, the use of conventional ultrasound could provide useful information about trypsin-induced progressive PG depletion in AC. USS and UAA are related to the degeneration of $\mathrm{AC}$ and therefore are two indicators of the tissue PG content depletion. These results may provide some valuable information for the early diagnosis of $\mathrm{AC}$ diseases.

This work was supported by the National Natural Science Foundation of China (Grant No. 31170896) and State Key Laboratory of Software Development Environment (Grant No. SKLSDE-2011ZX-11).

1 Mow V C, Ratcliffe A, Poole A R. Cartilage and diarthrodial joints as paradigms for hierarchical materials and structures. Biomaterials, 1992, 13: 67-97

2 Mow V C, Gu W Y, Chen F H. Structure and function of articular cartilage and meniscus. In: Basic Orthopaedic Biomechanics and Mechano-biology. 3rd ed. Mow V C, Huiskes R, eds. Philadelphia: Lippincott Williams \& Wilkins, 2005

3 Wang Q. Ultrasonic characterization of transient and inhomogeneous swelling behavior and progressive degeneration of articular cartilage. Dissertation for PhD Degree. Hong Kong: Hong Kong Polytechnic University, 2007

4 Mow V C, Guo X E. Mechano-electrochemical properties of articular cartilage: their inhomogeneities and anisotropies. Annu Rev Biomed Eng, 2002, 4: 175-209

5 Brandt K, Doherty M, Lohmander S. Osteoarthritis Cartilage. Oxford: Oxford University Press, 1998

6 Bland Y S, Ashhurst D E. Development and ageing of the articular cartilage of the rabbit knee joint: distribution of the fibrillar collagens. Anat Embryol, 1996, 194: 607-619

7 Weiss C, Mirow S. An ultrastructural study of osteoarthritic changes in the articular cartilage of human knees. Bone Joint Surg, 1972, 54: 954-972

8 Boven F, Bellemans M A, Geurts J, et al. The value of computed tomography scanning in chondromalacia patellae. Skeletal Radiol, 1982, 8: 183-185

9 Ihara H. Double-contrast CT arthrography of the cartilage of the patellofemoral joint. Clin Orthop, 1985, 198: 50-55

10 Gagliardi J A, Chung E M, Chandnani V P, et al. Detection and staging of chondromalacia patellae: relative efficacies of conventional MR imaging, MR arthrography, and CT arthrography. AJR Am J Roentgenol, 1994, 163: 629-636

11 Burstein D, Bashir A, Gray M L. MRI techniques in early stages of cartilage disease. Invest Radiol, 2000, 35: 622-638

12 Nieminen M T, Toyras J, Rieppo J, et al. Quantitative MR microscopy of enzymatically degraded articular cartilage. Magn Reson Med, 2000, 43: 676-681

13 Herrmann J M, Pitris C, Bouma B E, et al.. High resolution imaging of normal and osteoarthritic cartilage with optical coherence tomography. J Rheumatol, 1999, 26: 627-635

14 Legare A, Garon M, Binette F, et al. Evaluation of cartilage quality using streaming potential maps. Transact ICRS, 2000, 3: 89

15 Disler D G, Raymond E, May D A, et al. Articular cartilage defects: In vitro evaluation of accuracy and interobserver reliability for detection and grading with US. Radiology, 2000, 215: 846-851

16 Nieminen H J, Toyras J, Rieppo J, et al. Real-time ultrasound analysis of articular cartilage degradation in vitro. Ultrasound Med Biol, 2002, 28: 519-525

17 Niu H J, Wang Q, Zheng Y P, et al. A new method for computing the uniaxial modulus of articular cartilages using modified inhomogeneous triphasic model. Acta Mech Sin, 2010, 26: 121-126

18 Agemura D H, OBrien W D, Olerud J E, et al. Ultrasonic propagation properties of articular cartilage at $100 \mathrm{MHz}$. J Acoust Soc Am, 1990, 87: 1786-1791

19 Cherin E, Saied A, Pellaumail B, et al. Assessment of rat articular cartilage maturation using 50-MHz quantitative ultrasonography. Osteoarthr Cartilage, 2001, 9: 178-186

20 Myers S L, Dines K, Brandt D A, et al. Experimental assessment by 
high frequency ultrasound of articular cartilage thickness and osteoarthritic changes. J Rheumatol, 1995, 22: 109-116

21 Suh J K F, Youn I, Fu F H. An in situ calibration of an ultrasound transducer: a potential application for an ultrasonic indentation test of articular cartilage. J Biomech, 2001, 34: 1347-1353

22 Fortin M, Buschmann M D, Bertrand M J, et al. Dynamic measurement of internal solid displacement in articular cartilage using ultrasound backscatter. J Biomech, 2003, 36: 443-447

23 Hattori K, Ikeuchi K, Morita Y, et al. Quantitative ultrasonic assessment for detecting microscopic cartilage damage in osteoarthritis. Arthritis Res Ther, 2005, 7: 38-46

24 Hattori K, Takakura Y, Ishimura M, et al. Differential acoustic properties of early cartilage lesions in living human knee and ankle joints. Arthritis Rheum, 2005, 52: 3125-3131

25 Zheng Y P, Shi J, Qin L, et al. Dynamic depth-dependent osmotic swelling and solute diffusion in articular cartilage monitored using real-time ultrasound. Ultrasound Med Biol, 2004, 30: 841-849

26 Zheng Y P, Niu H J, Mak A F T, et al. Ultrasonic measurement of depth-dependent transient behaviors of articular cartilage under compression. J Biomech, 2005, 38: 1830-1837

27 Joiner G A, Bogoch E R, Pritzker K P, et al. High frequency acoustic parameters of human and bovine articular cartilage following experimentally-induced matrix degradation. Ultrasound Imag, 2001, 23: 106-116

28 Zheng Y P, Mak A F T, Lau K P, et al. An ultrasonic measurement for in vitro depth-dependent equilibrium strains of articular cartilage in compression. Phys Med Biol, 2002, 7: 3165-3180

29 Laasanen M S, Toyras J, Hirvonen J, et al. Novel mechano-acoustic technique and instrument for diagnosis of cartilage degeneration. Physiol Meas, 2002, 23: 491-503
30 Laasanen M S, Saarakkala S, Toyras J, et al. Ultrasound indentation of bovine knee articular cartilage in situ. J Biomech, 2003, 36: $1259-1267$

31 Wang Q, Zheng Y P, Guo X, et al. Progressive trypsin digestion and serum inhibition in articular cartilage monitored using highfrequency ultrasound in situ. In: Proceedings of the 30th Annual International Conference of the IEEE Engineering in Medicine and Biology Society, Vancouver, Canada, 2008. 2169-2172

32 Zheng Y P, Bridal S L, Shi J, et al. High resolution ultrasound elastomicroscopy imaging of soft tissues: System development and feasibility. Phys Med Biol, 2004, 49: 3925-3938

33 Toyras J, Laasanen M S, Saarakkala S, et al. Speed of sound in normal and degenerated bovine articular cartilage. Ultrasound Med Biol, 2003, 29: 447-454

34 Nieminen H J, Saarakkala S, Laasanen M S, et al. Ultrasound attenuation in normal and spontaneously degenerated articular cartilage. Ultrasound Med Biol, 2004, 30: 493-500

35 Wang Q, Zheng Y P, Qin L, et al. Real-time ultrasonic assessment of progressive proteoglycan depletion in articular cartilage. Ultrasound Med Biol, 2008, 34: 1085-1092

36 Pellaumail B, Watrin A, Loeuille D, et al. Effect of articular cartilage proteoglycan depletion on high frequency ultrasound backscatter. Osteoarthr Cartilage, 2002, 10: 535-541

37 Töyräs J, Rieppo J, Nieminen M T, et al. Characterization of enzymatically induced degradation of articular cartilage using high frequency ultrasound. Phys Med Biol, 1999, 44: 2723-2733

38 Huang N E, Long S R, Shen Z, et al. The empirical mode decomposition and the Hilbert spectrum for nonlinear and non-stationary time series analysis. Proc R Soc Lond A, 1998, 454: 903-995

Open Access This article is distributed under the terms of the Creative Commons Attribution License which permits any use, distribution, and reproduction in any medium, provided the original author(s) and source are credited. 